\title{
Tuning Organelle Specificity and Photodynamic Therapy Efficiency by Molecular Function Design
}

Zhiyang Liu, ${ }^{\dagger,+, \#}$ Hang Zou, ${ }^{\perp, \#}$ Zheng Zhao, ${ }^{\dagger}$ Pengfei Zhang, ${ }^{\dagger}$ Guogang Shan, ${ }^{\dagger}$ Ryan T. K. Kwok, '` Jacky W. Y. Lam, ${ }^{\dagger}$ Lei Zheng, ${ }^{* \perp}$ and Ben Zhong Tang ${ }^{*,+,+,}$

'Department of Chemistry, Hong Kong Branch of Chinese National Engineering Research Center for Tissue Restoration and Reconstruction, Division of Life Science and State Key Laboratory of Molecular Neuroscience, Institute of Molecular Functional Materials, Department Chemical and Biological Engineering and Institute for Advanced Study, The Hong Kong University of Science and Technology, Clear Water Bay, Kowloon, Hong Kong, China

${ }^{\sharp}$ HKUST Shenzhen Research Institute, No. 9 Yuexing 1st RD, South Area Hi-tech Park, Nanshan, Shenzhen 518057, China

${ }^{\perp}$ Department of Laboratory Medicine, Nanfang Hospital, Southern Medical University, Guangzhou, 510515, China.

'Center for Aggregation-Induced Emission, SCUT-HKUST Joint Research Institute, State Key Laboratory of Luminescent Materials and Devices, South China University of Technology, Guangzhou 510640, China

ABSTRACT: Efficient organic photosensitizers (PSs) have attracted much attention because of their promising applications in photodynamic therapy (PDT). However, guidelines on their molecular design are rarely reported. In this work, a series of PSs are designed and synthesized based on a triphenylamine-azafluorenone core. Their structure-property-application relationships are systematically studied. Cationization is an effective strategy to enhance the PDT efficiency of PSs. From the molecularly dispersed state to the aggregate state, the fluorescence and the reactive oxygen species generation efficiency of PSs with aggregation-induced emission (AIE) increase due to the restriction of the intramolecular motions and enhancement of intersystem crossing. Cationized mitochondrion-targeting PSs show higher PDT efficiency than that of nonionized ones targeting lipid droplets. The ability of AIE PSs to kill cancer cells can be further enhanced by combination of PDT with radiotherapy. Such results should trigger research enthusiasm for designing and synthesizing new AIE PSs with better PDT efficiency and new properties. 


\section{INTRODUCTION}

Conventional chemotherapy and radiotherapy remain the primary treatment methods in hospitals although they suffer from the resistance of cancer cells to drugs and radiation and severe side effects to result in unsatisfactory therapeutic effects and cancer recurrence. ${ }^{1}$ Thus, safe and effective therapies are urgently needed. Photodynamic therapy (PDT) has gained growing concern in clinical applications to cure various solid tumors, such as skin, esophagus and lung. ${ }^{2,3}$ PDT possesses several advantages such as high spatiotemporal precision, noninvasive nature, controllability, low toxicity, and repeatable treatment without initial resistance. ${ }^{4}$ Its mechanism is believed to be associated with the generation of reactive oxygen species (ROS) by photosensitizers (PSs) under light irradiation. ROS mainly includes two types. While superoxide anion $\left(\mathrm{O}_{2}{ }^{--}\right)$, hydroxyl radical $\left(\mathrm{OH}^{*}\right)$, peroxide $\left(\mathrm{O}_{2}{ }^{2-}\right)$ and so on fall into type $\mathrm{I}$, singlet oxygen $\left({ }^{1} \mathrm{O}_{2}\right)$ belongs to type II. These ROS could give rise to three main biochemical response to cure diseases comprising direct cell death via necrosis and/or apoptosis, microvascular shutdown and inflammatory and immune response. ${ }^{5}$ However, how to obtain a highly efficient and ideal PS for ROS generation and PDT lacks general guidelines, although it is known that enhancing intersystem crossing (ISC) from the excited singlet state $\left(\mathrm{S}_{1}\right)$ to the excited triplet $\left(\mathrm{T}_{1}\right)$ state and increasing the triplet state quantum yield and lifetime in theory are effective strategies to increase ROS generation. 6,7

Porphyrins and phthalocyanines are the most widely studied PSs with fast intersystem crossing and long triplet lifetime and have already applied in clinical applications. ${ }^{8,9}$ Nevertheless, these PSs usually aggregate in aqueous solutions due to their water solubility. This leads to the low fluorescence intensity and PDT efficiency because of aggregation-caused quenching (ACQ) effect, that makes image-guided PDT difficult to be achieved. To solve this problem, some water-soluble and non-aggregating PSs were synthesized. ${ }^{10}$ However, such approach greatly increased the cost of material preparation. In recent years, a new kind of PSs with aggregation-induced emission (AIE) characteristics has been developed by our group and others. ${ }^{11-19}$ The AIE PSs show not only improved fluorescence intensity but also efficient ROS generation capacity in 
aggregates or nanoparticles. These properties make them perfect candidates for imageguided PDT. However, the structure-performance relationship of AIE PSs for PDT is lesser studied than that of traditional ACQ PSs. Thus, besides molecular structures, the mechanisms for efficient ROS generation need to be deciphered.

ROS shows a short intracellular lifetime and its diffusion length $\left(<50 \mathrm{~nm}\right.$ for $\left.{ }^{1} \mathrm{O}_{2}\right)$ is much shorter than the diameters $(10-100 \mu \mathrm{m})$ of human cancer cells. ${ }^{20}$ Thus, the PSs are better to possess the capability to target specific subcellular structures, such as mitochondrion, lysosome, plasma membrane to achieve better PDT effect. ${ }^{21-23}$ Among the organelles, mitochondria are believed to be the ideal ones as they are important stations of energy conversion and oxidative metabolism. ${ }^{24,25}$ On the other hand, since molecular structures of PSs for targeting different organelles are different. Thus, even these PSs have similar ROS generation capacity, it is hard to realize the effect of organelle for their PDT efficiency under the same experimental conditions.

To partly illustrate these issues and deepen the understanding of AIE PSs, we designed and synthesized four new-style PSs, named TPAN, TPAPy, TPANPF 6 and $\mathrm{TPAPyPF}_{6}$ in this work with a common core forming by electron-donating triphenylamine and electron-withdrawing azafluorenone. Morpholine and different substituents including dimethylaniline, pyridine, trimethylphenylammonium hexafluorophosphate and 1-methylpyridin-1-ium hexafluorophosphate were attached to their periphery (Figure 1A). As, azafluorenone and morpholine groups have been found to show biological characteristics. ${ }^{26}$ The synthetic molecules are anticipated to find potential biological applications. The rational design principles and the functional mechanism of the PSs were explored by comparing their ROS generation capacity, organelle targeting ability and PDT efficiency. 

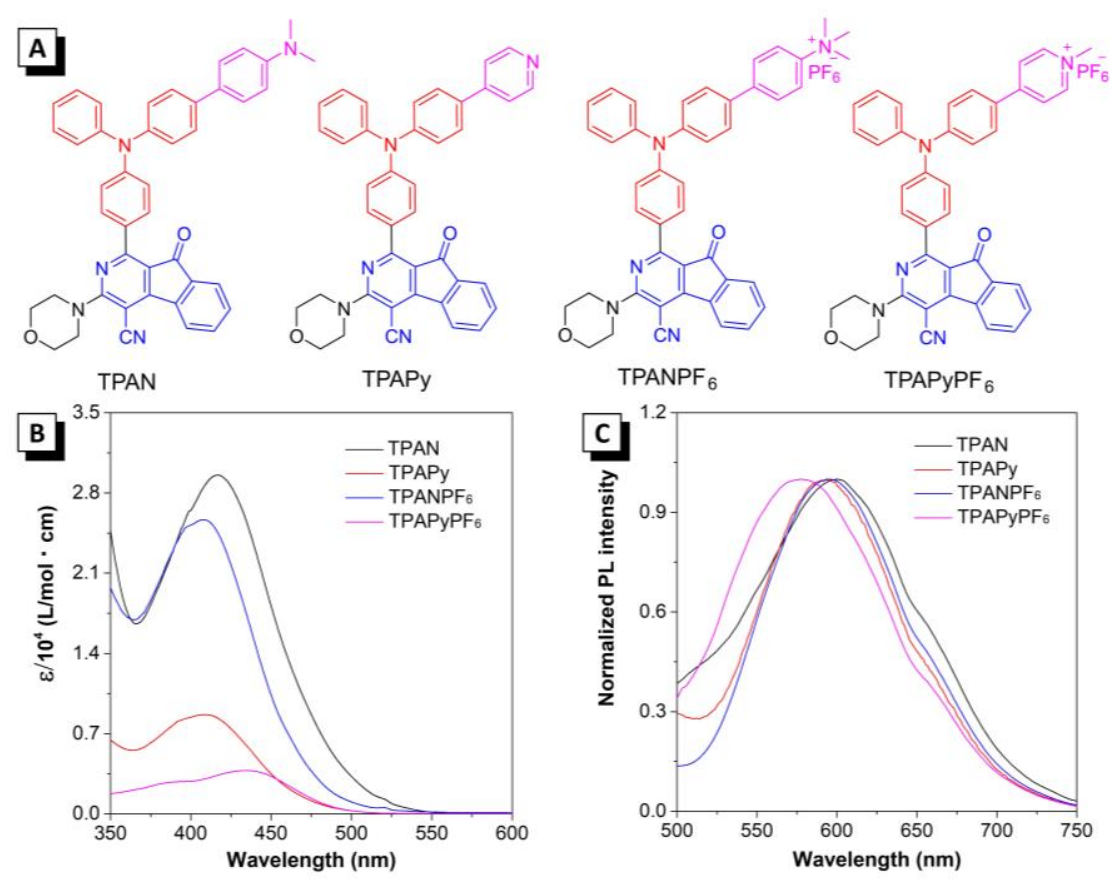

Figure 1. (A) Molecular structures of the target materials. (B) molar absorption coefficient of TPAN, TPAPy, TPANPF 6 , and TPAPyPF 6 in THF solution. (C) Normalized PL spectra of TPAN, TPAPy, $\mathrm{TPANPF}_{6}$, and $\mathrm{TPAPyPF}_{6}$ in dioxane solution.

\section{RESULTS AND DISCUSSION}

The photophysical properties of the four compounds were first studied, Figure 1 shows their UV-vis absorption and photoluminescence (PL) spectra. TPAN shows a longer cut-off absorption wavelength and stronger molar absorption coefficients than other compounds in tetrahydrofuran solution. On the other hand, the molar absorption coefficients of the ionized compounds, i.e., TPANPF 6 and $\mathrm{TPAPyPF}_{6}$ are inferior than their corresponding nonionized counterparts (TPAN and TPAPy). TPAPyPF 6 absorbs lesser light than other molecules. Their PL spectra spread a similar wavelength range from $500 \mathrm{~nm}$ to $750 \mathrm{~nm}$ and are peaked at about $600 \mathrm{~nm}$ in dioxane. Surprisingly, their solid PL spectra are distinctly different. TPAN exhibits a prominent solvatochromic effect. While it offers only weak emission in non-polar solvents, it even becomes nonemissive in polar solvents and solid state due to the strong twisted intramolecular charge transfer. ${ }^{27}$ This suggests that it is an ACQ molecule. On the other hand, TPANPF6 exhibits a redder PL $(617 \mathrm{~nm})$ than TPAPy $(579 \mathrm{~nm})$ and TPAPyPF $6(571 \mathrm{~nm})$ in the solid state. As shown in Figure 2, TPAPy, $\mathrm{TPANPF}_{6}$ and $\mathrm{TPAPyPF}_{6}$ are all AIE-active, 
as verified by their enhanced PL intensity in tetrahydrofuran (THF)/water or dimethylsulfoxide (DMSO)/water mixtures with high water fractions. Through the PL analysis, TPANPF 6 shows the phenomenon of ionization-caused AIE. ${ }^{28}$
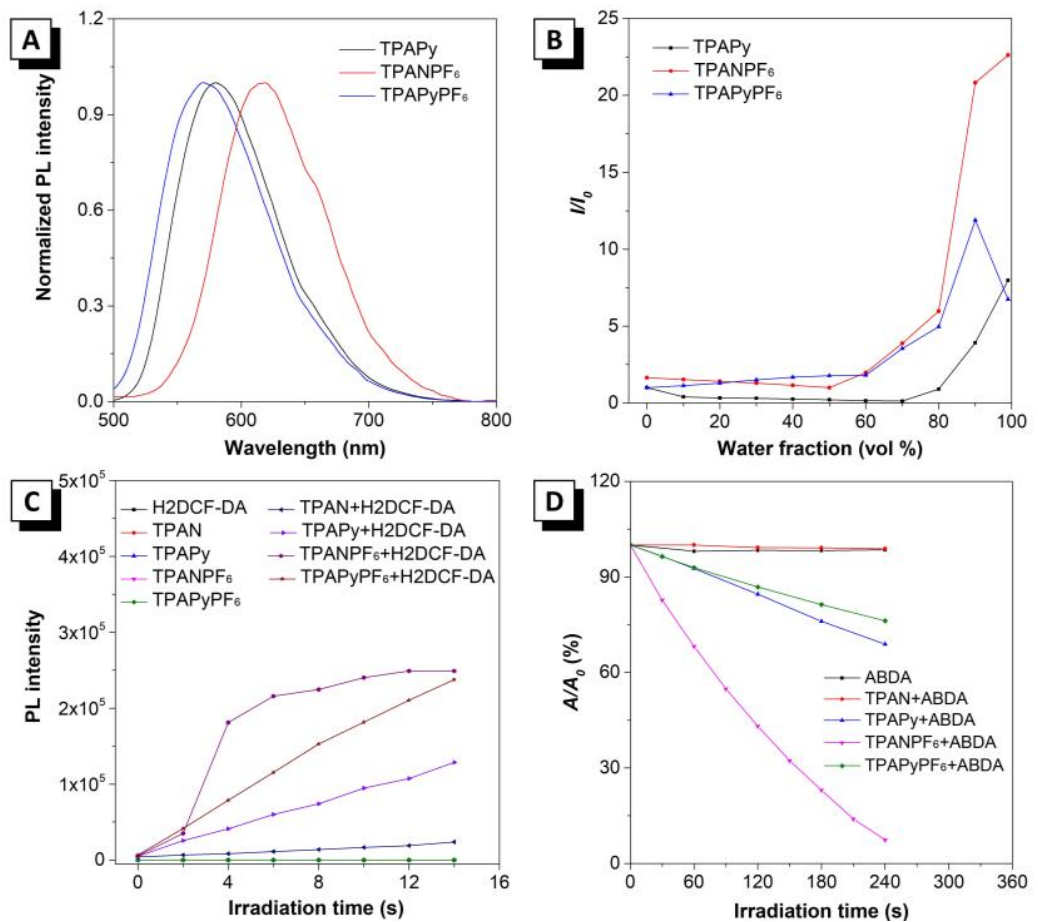

Figure 2. (A) Normalized PL spectra of TPAPy, $\mathrm{TPANPF}_{6}$, and TPAPyPF 6 in the solid state. (B) Plot of relative PL intensity $\left(I / I_{0}\right)$ vs the composition of the THF/water (TPAPy) or DMSO/water (TPANPF 6 and $\mathrm{TPAPyPF}_{6}$ ) mixtures. (C) Change of PL intensity at $525 \mathrm{~nm}$ of H2DCF-DA in the presence or absence of four compounds in PBS and light irradiation for different time. (D) The decomposition rates of ABDA in the presence or absence of four compounds and light irradiation, where $A_{0}$ and $A$ are the absorbance of ABDA at $378 \mathrm{~nm}$ before and after irradiation, respectively.

To evaluate the performance of the four PSs to produce ROS, a commercial indicator called 2',7'-dichlorodihydrofluorescein diacetate (H2DCF-DA) was employed under white light irradiation $\left(4.2 \mathrm{~mW} / \mathrm{cm}^{2}\right)$ in phosphate-buffered saline (PBS) for its ability to fluoresce at about $525 \mathrm{~nm}$ after being oxidized by ROS. ${ }^{29}$ Results shown in Figure 2C suggest that the ROS generation capacity is in sequence of $\mathrm{TPANPF}_{6}>\mathrm{TPAPyPF}_{6}>\mathrm{TPAPy}>\mathrm{TPAN}$ Because $\mathrm{TPANPF}_{6}$ forms nanoparticles (NPs) in PBS with fast ROS generation rate, the fluorescence of H2DCF-DA reaches its plateau value in only $\sim 6 \mathrm{~s}$. The NPs of its nonionized parent form, i.e. TPAN with ACQ effect produce only little ROS in PBS. Similarly, the ROS generation capacity of ionized TPAPyPF 6 NPs is higher than TPAPy NPs. It is generally known that H2DCFDA is capable of responding to many types of ROS including type I and type II. For 
type I, the excited triplet state of PS transfers an electron to a substrate to produce radicals or radical ions such as $\mathrm{O}_{2}{ }^{\circ}, \mathrm{OH}^{*}, \mathrm{O}_{2}{ }^{2-}$. For type II, the excited triplet state of PS interacts with triplet oxygen $\left({ }^{3} \mathrm{O}_{2}\right)$ by an energy transfer pathway to yield highly reactive ${ }^{1} \mathrm{O}_{2}$ (Figure 3C). ${ }^{6,30}$ That is generally identified as the primary species for PDT due to its higher diffusibility and stronger interaction with body substrates than radical species. ${ }^{20,31}$ Thus, it is essential to measure the ${ }^{1} \mathrm{O}_{2}$ generation of the PSs. Commercially available material called 9,10-anthracenediyl-bis-(methylene)-dimalonic acid (ABDA) is a ${ }^{1} \mathrm{O}_{2}$ indicator and can detect the ${ }^{1} \mathrm{O}_{2}$ generation of a material by monitoring its absorption change at $378 \mathrm{~nm}$. The ${ }^{1} \mathrm{O}_{2}$ generation ability of the four PSs is different and is in the order of $\mathrm{TPANPF}_{6}>\mathrm{TPAPy}>\mathrm{TPAPyPF}_{6}>\mathrm{TPAN}$ (Figure 2D). Whereas the absorption of aqueous solutions of ABDA with TPAPy, TPANPF 6 or TPAPyPF 6 NPs gradually decreases with the extension of the white light irradiation $\left(4.2 \mathrm{~mW} / \mathrm{cm}^{2}\right)$ time, almost no absorption change was observed in TPAN NPs. The consumption rate of ABDA was calculated to be $0.7,2.32$ and $0.6 \mathrm{nmol} / \mathrm{min}$ for TPAPy, TPANPF 6 and TPAPyPF6 under the same conditions, respectively. The ROS and ${ }^{1} \mathrm{O}_{2}$ generation abilities of TPAPy and TPAPyPF 6 NPs are in inconsistent order (Figure 2C and 2D), suggesting that TPAPyPF 6 produces not only ${ }^{1} \mathrm{O}_{2}$ but also type I ROS. To explain this, sodium azide $\left(\mathrm{NaN}_{3}\right)$ as a ${ }^{1} \mathrm{O}_{2}$ quencher was added to the $\mathrm{ABDA}$ solution in the presence of TPAPy, TPANPF 6 or TPAPyPF 6 NPs $^{32}$ The absorption change of ABDA with TPAPy and $\mathrm{NaN}_{3}(30 \mathrm{mg} / \mathrm{mL})$ in water was negligible in the presence of white light irradiation. However, the absorption of ABDA with $\mathrm{TPANPF}_{6}$ or TPAPyPF 6 gradually declined with prolonged irradiation time even at a high concentration of $\mathrm{NaN}_{3}(240 \mathrm{mg} / \mathrm{mL})$. This suggests that ionized TPANPF 6 and TPAPyPF 6 could generate different types of ROS and the nonionized TPAPy produces only the ${ }^{1} \mathrm{O}_{2} .{ }^{33}$ To further prove this, dihydroethidium (DHE) was used as a sensitive $\mathrm{O}_{2}{ }^{--}$probe to assess the $\mathrm{O}_{2}{ }^{\bullet-}$ generation in HeLa cells by flow cytometry. ${ }^{34,35}$ Results show that the fluorescence of HeLa cells with DHE becomes stronger in the presence of TPANPF6 or TPAPyPF6 under light irradiation. Clearly, $\mathrm{TPANPF}_{6}$ and $\mathrm{TPAPyPF}_{6}$ could generate a small amount of $\mathrm{O}_{2}{ }^{\circ-}$, which is the precursor of $\mathrm{OH}^{\cdot}$ and $\mathrm{H}_{2} \mathrm{O}_{2} \cdot{ }^{36}$ Therefore, the ROS generation ability of TPAPyPF 6 is higher than TPAPy when H2DCF-DA is used as an indicator. 
The ROS generation ability of the four PSs seems to exert great change by only a subtle functional group variation. According to previous literatures, promoting ISC from $S_{1}$ to $T_{1}$ is conducive to enhancing the photosensitization efficiency of PSs by increasing the spin-orbit coupling (SOC) and decreasing the $\Delta E_{S T}$ based on the perturbation theory and Marcus semiclassical method ${ }^{37}$ Hence, we first calculated the highest occupied molecular orbital (HOMO), lowest unoccupied molecular orbital (LUMO), SOC and $\Delta E S T$ of the PSs by density functional theory. ${ }^{38}$ Except TPAPyPF 6 , TPAN, TPAPy and TPANPF 6 show distinct orbital distributions of HOMO and LUMO. The $\Delta E_{S T}$ (the energy gap between $S_{1}$ state and $T_{1}$ state) values are calculated to be $0.0909,0.1645$ and $0.1831 \mathrm{eV}$ for TPAN, TPAPy and TPANPF 6 , respectively. The $\Delta E_{S T}$ of TPAPyPF 6 is $0.5155 \mathrm{eV}$ but its $\mathrm{S}_{1}$ energy is higher than $\mathrm{T}_{2}\left(\Delta E_{S_{I} T_{2}}=0.2783 \mathrm{eV}\right)$, $\mathrm{T}_{3}\left(\Delta E_{S_{T_{3}}}=0.02 \mathrm{eV}\right)$ and $\mathrm{T}_{4}\left(\Delta E_{S_{I} T_{4}}=0.0076 \mathrm{eV}\right)$ value, which could reduce the energy gap between the singlet state and the triplet state to improve the ISC. On the other hand, the SOC effect is also a principal element for ISC. The calculated SOC constants of the present PSs are all very small and fall in a similar range $\left(\sim 0.1-3 \mathrm{~cm}^{-1}\right)$ for most reported organic materials so far. ${ }^{39,40}$ Thus, decreasing $\Delta E_{S T}$ is generally considered to be the main factor for enhancing ISC of organic materials and boosting the ROS generation efficiency of PSs. However, such a reason cannot fully explain the difference in ROS generation capacity of the PSs in this work, especially for TPAN as its $\Delta E_{S T}$ is very small but it barely produces ROS. This suggests the existence of other factors that collectively affect the ROS generation. Thereinto, as mentioned above, the large difference in molar absorption coefficient of PSs should play also an important role due to the disparity of the absorbed total energy at the same molar. Furthermore, the aggregation of some AIEgens can strengthen the energy matches between the excited singlet and the triplet states by energy spitting to lead to heighten the ISC rate and prolong the lifetime of the triplet state. ${ }^{41}$ Therefore, the aggregation of AIE PSs should be able to improve the ROS generation efficiency. 

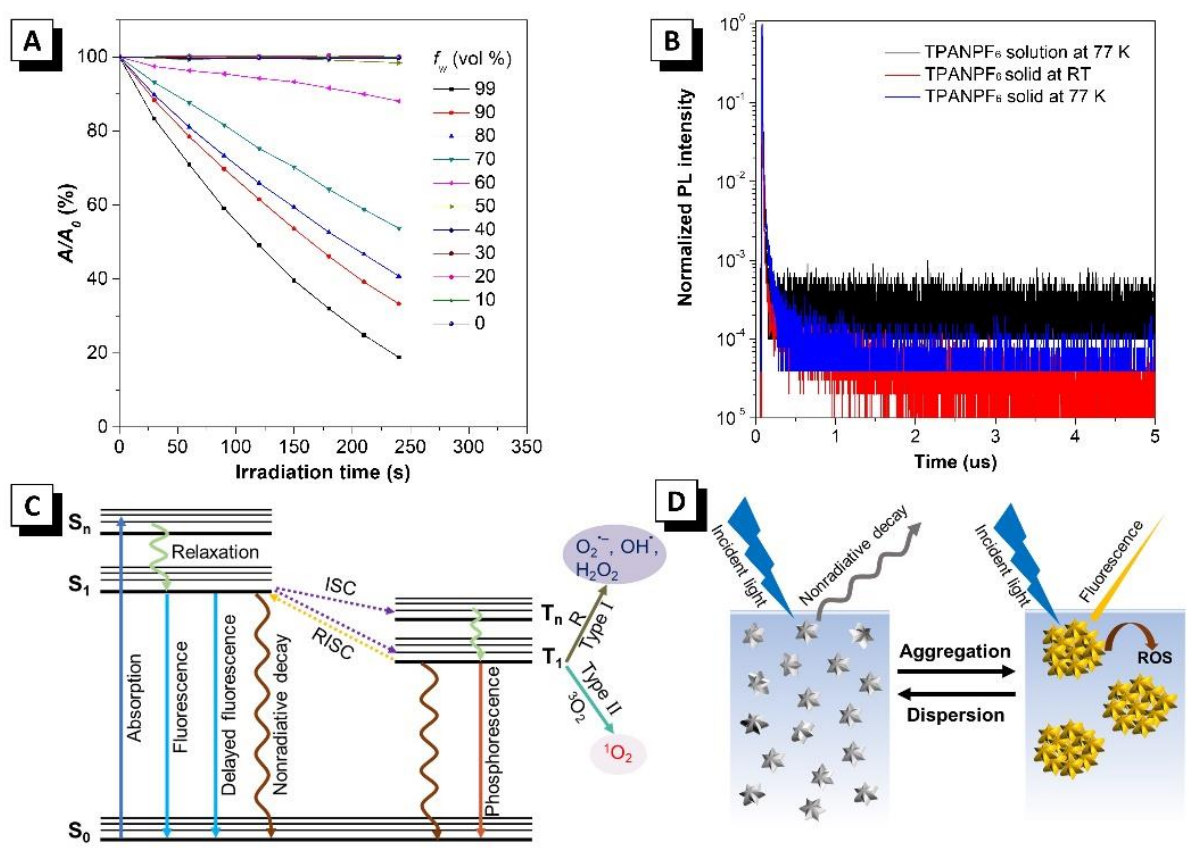

Figure 3. (A) The decomposition of ABDA in the presence of $\mathrm{TPANPF}_{6}$ under light irradiation in DMSO/water mixtures with different water fractions $\left(f_{w}\right)$. (B) Delayed PL decay curves of $\mathrm{TPANPF}_{6}$ under different conditions. (C) Schematic diagram of the basic mechanisms of photodynamic therapy. (D) The main way of energy consumption of TPANPF 6 in the aggregate and dispersed states under light irradiation.

To verify this, the absorption changes of ABDA in the presence of TPAN, TPAPy, $\mathrm{TPANPF}_{6}$ or $\mathrm{TPAPyPF}_{6}$ were measured under light irradiation in different organic solvents including DMSO, THF, methanol and chloroform, respectively. Again, TPAN scarcely generates ${ }^{1} \mathrm{O}_{2}$ in both the dissolved state and nanoparticles. This suggests that the excited state mainly consumes its energy through the nonradiative decay pathway owing to the strong ICT effect of the TPAN molecule. AIE-active TPAPy, TPANPF6 and TPAPyPF 6 also produce almost no ${ }^{1} \mathrm{O}_{2}$ when they are molecularly dissolved in organic solvent as the active intramolecular motions in solution make the excitons to relax nonradiatively to lessen the ISC process. Thus, the PL and ${ }^{1} \mathrm{O}_{2}$ generation efficiency of the three AIE PSs are closely associated with the AIE mechanism and become high when the nonradiative decay is suppressed. We chose TPANPF6 with a fast ${ }^{1} \mathrm{O}_{2}$ generation rate as an example for further discussion. As shown in Figure $3 \mathrm{~A}$, the absorption change of $\mathrm{ABDA}_{\text {with }} \mathrm{TPANPF}_{6}$ under light irradiation exhibits a same trend with the fluorescence change of $\mathrm{TPANPF}_{6}$ in $\mathrm{DMSO} /$ water mixtures with 
different water fractions. TPANPF 6 begins to produce ${ }^{1} \mathrm{O}_{2}$ and shows enhanced fluorescence when the water content exceeds $\geq 50 \%$ because the aggregation of TPANPF 6 occurs as confined by dynamic light scattering measurement. The fluorescence and ${ }^{1} \mathrm{O}_{2}$ generation of TPANPF 6 become gradually higher with increasing the water fraction as aggregates gradually evolve from loose ones to compact ones. It indicates that the aggregation of AIE PSs contributes to enhancing both ROS generation efficiency and fluorescence intensity owing to the AIE mechanism to decrease the nonradiative decay. The PL decay curve of $\mathrm{TPANPF}_{6}$ in the solid state at room temperature (RT) reveals a delayed fluorescence (DF) lifetime ( $\tau=188.36 \mathrm{~ns})$ but no such signal was detected in solution. At $77 \mathrm{~K}$, the PL lifetime becomes short in solid state $(\tau=26.26 \mathrm{~ns})$ or in solution $(\tau=12.93 \mathrm{~ns})$ (Figure 3B). TPAPy and TPAPyPF6 display also a similar phenomenon, demonstrating that they may have the thermally activated delayed fluorescence in the solid state in virtue of the small $\Delta E_{S T}$ (Figure 3C). ${ }^{42}$ This also provides a compelling evidence for the phenomenon of aggregationenhanced ISC in the three AIE PSs. The order of PL lifetime of the three AIE PSs is also consistent with the order of ROS generation efficiency. TPAN shows the ACQ effect and shows only a long lifetime in the solid state at $77 \mathrm{~K}$, suggesting that the energy of the excited energy is consumed mainly through the nonradiative decay pathway at RT. Overall, the AIE PSs demonstrate aggregation-induced ROS generation characteristics due to the suppression of the nonradiative decay to facilitate ISC at room temperature and the ROS generation rate of PSs depends on the combined effect of various factors in vitro. 


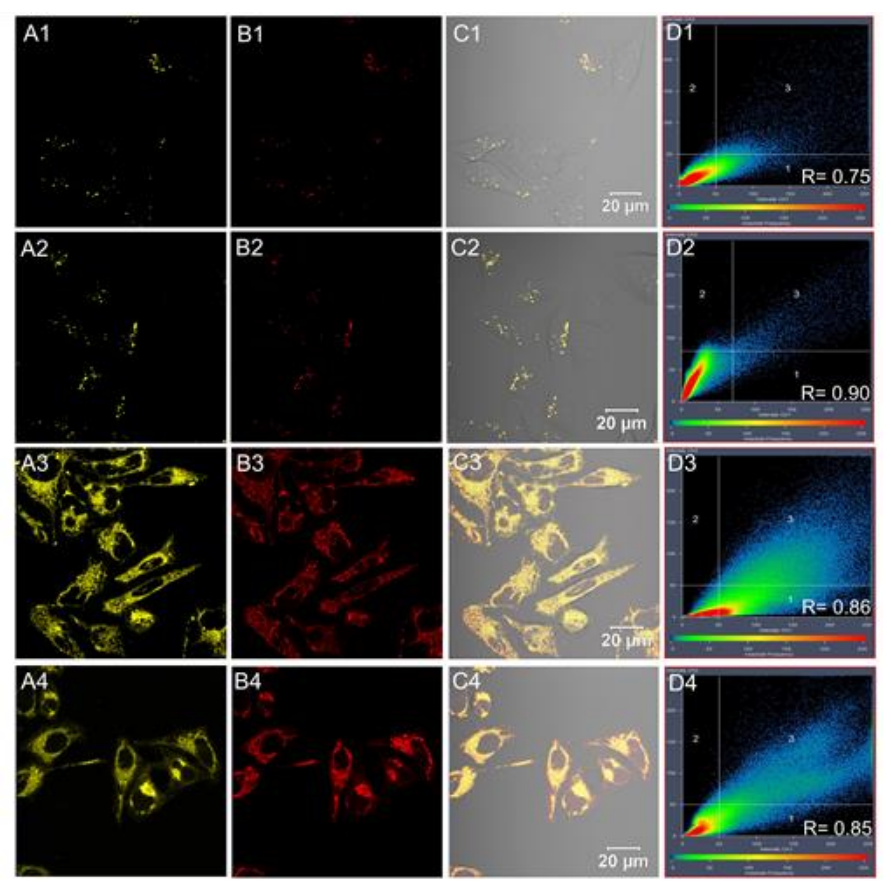

Figure 4. Co-localization imaging of HeLa cells stained with Nile Red, MitoTracker Deep Red (MTDR) and the synthesized molecules. (A and B) confocal images of HeLa cells stained with (A1) TPAN, (A2) TPAPy, (A3) TPANPF 6 , (A4) TPAPyPF 6 , (B1 and B2) Nile Red, and (B3 and B4) MitoTracker Deep Red. (C1-C4) Merged images of panels A and B. (D1-D4) A scatter plot indicating the correction coefficient between panel A and B. $\lambda_{\text {ex }}: 405 \mathrm{~nm}$. Concentration: $10 \mu \mathrm{M}$ (TPAN, TPAPy, TPANPF $_{6}$ and TPAPyPF 6 ), 2 ng/mL (Nile Red), $50 \mathrm{nM}$ (MTDR). Scale bar: $20 \mu \mathrm{m}$.

Anyhow, the intracellular environment is complex with diverse organelles and compounds. Hence, there may be new factors affecting the ROS generation efficiency of PSs to disturb the image-guided PDT efficiency in cells. First, the organelle specificity of the present PSs was evaluated by the confocal laser scanning microscope. TPAN is encapsulated by amphiphilic F127 to obtain NPs with good water dispersibility due to its strong hydrophobic character and poor polar solvent solubility in polar solvent. Simultaneously, the NPs also endow ACQ TPAN with the luminescent imaging property. AIE TPAPy, TPANPF 6 and TPAPyPF6 were dissolved in DMSO and the resulting solution were added directly to the cell culture medium to form NPs for imaging. As illustrated in Figure 4, the colocalization experiments were performed by staining HeLa cells with the synthesized PSs, Nile Red (the lipid droplet staining agent) and MitoTracker Deep Red (MTDR, the mitochondrion-staining agent), respectively. It reveals that the nonionized TPAN and TPAPy principally stain LDs due 
to their strong lipophilic character $(\operatorname{LogP}>7)$, while the ionized $\mathrm{TPANPF}_{6}$ and TPAPyPF 6 both specifically target the mitochondria owing to the electrostatic attraction with the mitochondrial membrane potential (MMP). ${ }^{43,44}$ High Pearson's correlation coefficients $(\mathrm{R}=0.75-0.90)$ are obtained between the PSs and the commercial probes, indicating the excellent organelle specificity of the present new molecules. The cytotoxicity of the PSs at different concentrations was tested by using 3-(4,5-dimethyl2-thiazolyl)-2,5-diphenyltetrazolium bromide (MTT) assay. Results show that the AIE PSs have negligible cytotoxicity even at a concentration of up to $40 \mu \mathrm{M}$ for $24 \mathrm{~h}$, demonstrating their outstanding biocompatibility.
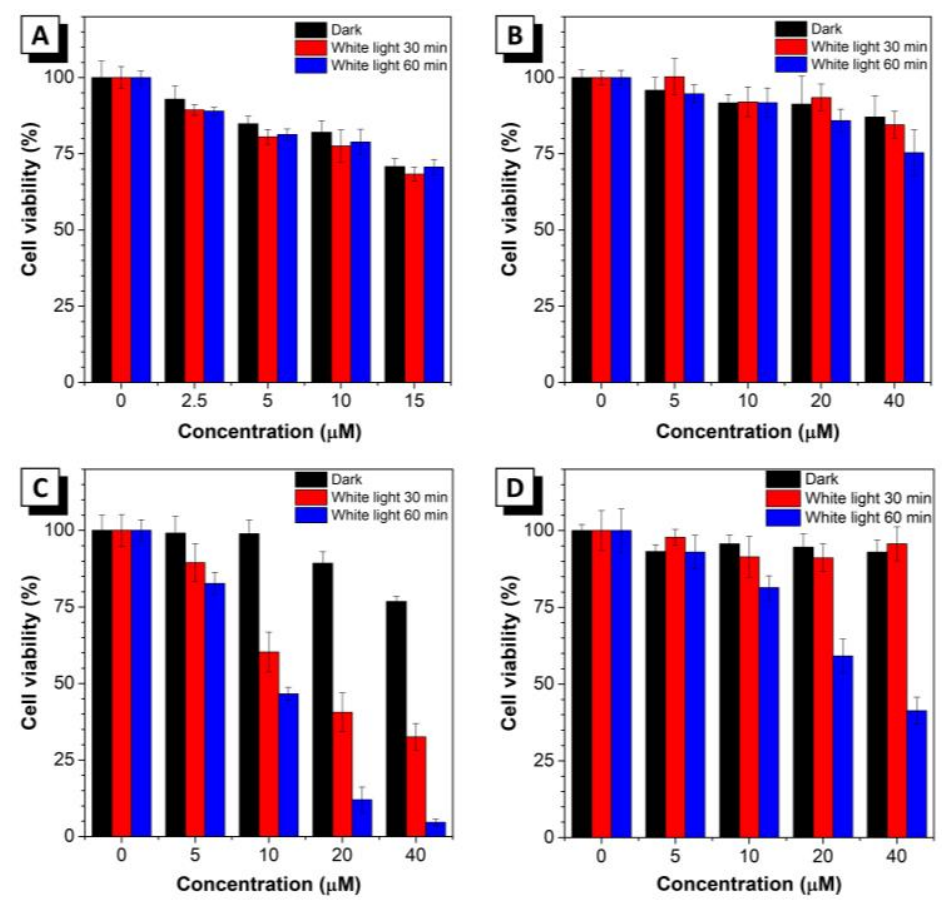

Figure 5. Cell viability of HeLa cells stained with different concentrations of (A) TPAN, (B) TPAPy, (C) $\mathrm{TPANPF}_{6}$, (D) TPAPyPF 6 under dark or under white-light irradiation.

Subsequently, the PDT effect was studied by incubating HeLa cells with various concentrations of PSs under weak white light $\left(4.2 \mathrm{~mW} / \mathrm{cm}^{2}\right)$ exposure for different time. As depicted in Figure 5, the PSs show completely diverse PDT efficiency. TPAN only exhibits negligible cytotoxicity without PDT effect both in the dark and under light irradiation because it produces little ROS both in vitro and in cells. Interestingly, TPAPy hardly shows any PDT effect in HeLa cells even at a concentration of up to 40 $\mu \mathrm{M}$ for 60 min illumination, although it can quickly generate ${ }^{1} \mathrm{O}_{2}$ in vitro in a rate much 
faster than that of TPAPyPF 6 . On the other hand, TPAPyPF 6 displays a clear PDT effect under the same conditions and causes a decrease of cell viability by about $45 \%$. The main difference between TPAPy and TPAPyPF 6 in HeLa cells is the organelle they target. It seems that PSs that target mitochondria show more effective PDT effect than those that are targeting lipid droplets in HeLa cells. As stated before, mitochondria are the power stations of cells and play major roles in energy metabolism and cellular signaling. They are also considered as the main organelles for PDT-induced cell apoptosis or necrosis. On the other hand, the neutral environment inside lipid droplets may reduce the aggregation of nonionized PSs targeting to decrease the ${ }^{1} \mathrm{O}_{2}$ generation. Therefore, the mitochondrion-targeting $\mathrm{TPANPF}_{6}$ with the fast ${ }^{1} \mathrm{O}_{2}$ generation exerts the best PDT efficiency than other three PSs. The viability of HeLa cells decreases to $\sim 40 \%$ at a TPANPF 6 concentration of $20 \mu \mathrm{M}$ and $30 \mathrm{~min}$ illumination. At $60 \mathrm{~min}$, the cell viability drops to $\sim 10 \%$. The dependence of cell viability on the photosensitizer dose and illumination time is in line with the characteristics of PDT. Consequently, the ionized TPANPF6 could be an effective PS to eliminate cancer cells by PDT.

\section{CONCLUSIONS}

In summary, four PSs based on triphenylamine-azafluorenone core are synthesized and found to exhibit different photophysical properties and biological applications even they show only a subtle functional group. Cationization is found to be an effective strategy to enhance the ROS generation and PDT efficiency of PSs. The AIE PSs exhibit the concurrent increase of fluorescence intensity and ROS generation from the molecular dispersion state to the aggregate state due to the AIE mechanism and enhancement of ISC. This helps to easily achieve image-guided PDT without complex chemical synthesis. On the other hand, cationized mitochondrion-targeting PSs show higher PDT efficiency than that of nonionized PSs that target lipid droplets. This work thus provides a new perspective on the molecular design of PSs to improve the PDT efficiency. 


\section{AUTHOR INFORMATION}

\section{Corresponding Authors}

*E-mail: tangbenz@ust.hk (B. Z. Tang)

*E-mail: nfyyzhenglei@smu.edu.cn (L. Zheng)

\section{Author Contributions}

\#Z. Y. Liu and H. Zou contributed equally to this work.

\section{Notes}

The authors declare no competing financial interest.

\section{ACKNOWLEDGMENTS}

This work was financially supported by the National Science Foundation of China (21788102), the University Grants Committee of Hong Kong (AIE/P-03/08), the Research Grants Council of Hong Kong (16308016, C6009-17G and AHKUST605/16), the Innovation and Technology Commission (ITC-CNERC14SC01, ITCPD/17-9 and ITS/254/17), the National Key Research and Development program of China (2018YFE0190200) and Science and Technology Plan of Shenzhen (JCYJ20170818113530705 and JCYJ20170818113602462).

\section{REFERENCES}

(1) Karpuz, M.; Silindir-Gunay, M.; Ozer, A. Y. Current and Future Approaches for Effective Cancer Imaging and Treatment. Cancer Biother. Radiopharm. 2018, 33, 3951.

(2) Castano, A. P.; Mroz, P.; Hamblin, M. R. Photodynamic Therapy and Anti-Tumour Tmmunity. Nat. Rev. Cancer. 2006, 6, 535-545.

(3) Celli, J. P.; Spring, B. Q.; Rizvi, I.; Evans, C. L.; Samkoe, K. S.; Verma, S.; Pogue, 
B. W.; Hasan, T. Imaging and Photodynamic Therapy: Mechanisms, Monitoring, and Optimization. Chem. Rev. 2010, 110, 2795-2838.

(4) Zhou, Z.; Song, J.; Nie, L.; Chen, X. Reactive Oxygen Species Generating Systems Meeting Challenges of Photodynamic Cancer Therapy. Chem. Soc. Rev. 2016, 45, 65976626.

(5) Dolmans, D. E. J. G. J.; Fukumura, D.; Jain, R. K. Photodynamic Therapy for Cancer. Nat. Rev. Cancer. 2003, 3, 380-387.

(6) DeRosa, M. C.; Crutchley, R. J. Photosensitized Singlet Oxygen and Its Applications. Coord. Chem. Rev. 2002, 233-234, 351-371.

(7) Zhao, J.; Wu, W.; Sun, J.; Guo, S. Triplet Photosensitizers: from Molecular Design to Applications. Chem. Soc. Rev. 2013, 42, 5323-5351.

(8) Kou, J.; Dou, D.; Yang, L. Porphyrin Photosensitizers in Photodynamic Therapy and Its Applications. Oncotarget 2017, 8, 81591-81603.

(9) Wong, R. C. H.; Lo, P.-C.; Ng, D. K. P. Stimuli Responsive Phthalocyanine-Based Fluorescent Probes and Photosensitizers. Coord. Chem. Rev. 2019, 379, 30-46.

(10) Li, X.; Zheng, B.-D.; Peng, X.-H.; Li, S.-Z.; Ying, J.-W.; Zhao, Y.; Huang, J.-D.; Yoon, J. Phthalocyanines as Medicinal Photosensitizers: Developments in the Last Five Years. Coord. Chem. Rev. 2019, 379, 147-160.

(11) Zheng, Z.; Zhang, T.; Liu, H.; Chen, Y.; Kwok, R. T. K.; Ma, C.; Zhang, P.; Sung, H. H. Y.; Williams, I. D.; Lam, J. W. Y.; Wong, K. S.; Tang, B. Z. Bright Near-Infrared Aggregation-Induced Emission Luminogens with Strong Two-Photon Absorption, Excellent Organelle Specificity, and Efficient Photodynamic Therapy Potential. ACS Nano 2018, 12, 8145-8159.

(12) Zhang, T.; Li, Y.; Zheng, Z.; Ye, R.; Zhang, Y.; Kwok, R. T. K.; Lam, J. W. Y.; Tang, B. Z. In Situ Monitoring Apoptosis Process by a Self-Reporting Photosensitizer. J. Am. Chem. Soc. 2019, 141, 5612-5616.

(13) Wang, D.; Lee, M. M. S.; Xu, W.; Shan, G.; Zheng, X.; Kwok, R. T. K.; Lam, J. W. Y.; Hu, X.; Tang, B. Z. Boosting Non-Radiative Decay to Do Useful Work: Development of a Multi-Modality Theranostic System from an AIEgen. Angew. Chem. Int. Ed. 2019, 58, 5628-5632. 
(14) Wu, W.; Mao, D.; Xu, S.; Ji, S.; Hu, F.; Ding, D.; Kong, D.; Liu, B. High Performance Photosensitizers with Aggregation-Induced Emission for Image-Guided Photodynamic Anticancer Therapy. Mater. Horiz. 2017, 4, 1110-1114.

(15) Hu, F.; Mao, D.; Kenry; Cai, X.; Wu, W.; Kong, D.; Liu, B. A Light-Up Probe with Aggregation-Induced Emission for Real-Time Bio-orthogonal Tumor Labeling and Image-Guided Photodynamic Therapy. Angew. Chem. Int. Ed. 2018, 57, 1018210186.

(16) Wang, S.; Wu, W.; Manghnani, P.; Xu, S.; Wang, Y.; Goh, C. C.; Ng, L. G.; Liu, B. Polymerization-Enhanced Two-Photon Photosensitization for Precise Photodynamic Therapy. ACS Nano 2019, 13, 4742-4751.

(17) Li, M.; Gao, Y.; Yuan, Y.; Wu, Y.; Song, Z.; Tang, B. Z.; Liu, B.; Zheng, Q. C. One-Step Formulation of Targeted Aggregation-Induced Emission Dots for ImageGuided Photodynamic Therapy of Cholangiocarcinoma. ACS Nano 2017, 11, 39223932.

(18) Alifu, N.; Dong, X.; Li, D.; Sun, X.; Zebibula, A.; Zhang, D.; Zhang, G.; Qian, J. Aggregation-Induced Emission Nanoparticles as Photosensitizer for Two-Photon Photodynamic Therapy. Mater. Chem. Front. 2017, 1, 1746-1753.

(19) Sun, X.; Zebibula, A.; Dong, X.; Zhang, G.; Zhang, D.; Qian, J.; He, S. Aggregation-Induced Emission Nanoparticles Encapsulated with PEGylated Nano Graphene Oxide and Their Applications in Two-Photon Fluorescence Bioimaging and Photodynamic Therapy in Vitro and in Vivo. ACS Appl. Mater. Interfaces 2018, 10, 25037-25046.

(20) Ochsner, M. Photophysical and Photobiological Processes in the Photodynamic Therapy of Tumours. J. Photochem. Photobiol. B 1997, 39, 1-18.

(21) Kim, K. Y.; Jin, H.; Park, J.; Jung, S. H.; Lee, J. H.; Park, H.; Kim, S. K.; Bae, J.; Jung, J. H. Mitochondria-Targeting Self-Assembled Nanoparticles Derived from Triphenylphosphonium-Conjugated Cyanostilbene Enable Site-Specific Imaging and Anticancer Drug Delivery. Nano Res. 2018, 11, 1082-1098.

(22) Cai, X.; Mao, D.; Wang, C.; Kong, D.; Cheng, X.; Liu, B. Multifunctional Liposome: A Bright AIEgen-Lipid Conjugate with Strong Photosensitization. Angew. 
Chem. Int. Ed. 2018, 57, 16396-16400.

(23) Wang, D.; Su, H.; Kwok, R. T. K.; Hu, X.; Zou, H.; Luo, Q.; Lee, M. M. S.; Xu, W.; Lam, J. W. Y.; Tang, B. Z. Rational Design of a Water-Soluble NIR AIEgen, and Its Application in Ultrafast Wash-Free Cellular Imaging and Photodynamic Cancer Cell Ablation. Chem. Sci. 2018, 9, 3685-3693.

(24) Zielonka, J.; Joseph, J.; Sikora, A.; Hardy, M.; Ouari, O.; Vasquez-Vivar, J.; Cheng, G.; Lopez, M.; Kalyanaraman, B. Mitochondria-Targeted Triphenylphosphonium-Based Compounds: Syntheses, Mechanisms of Action, and Therapeutic and Diagnostic Applications. Chem. Rev. 2017, 117, 10043-10120.

(25) Zhang, D.; Wen, L.; Huang, R.; Wang, H.; Hu, X.; Xing, D. Mitochondrial Specific Photodynamic Therapy by Rare-Earth Nanoparticles Mediated Near-Infrared Graphene Quantum Dots. Biomaterials 2018, 153, 14-26.

(26) Tugrak, M.; Inci Gul, H.; Sakagami, H.; Gulcin, I.; Supuran, C. T. New Azafluorenones with Cytotoxic and Carbonic Anhydrase Inhibitory Properties: 2-Aryl4-(4-hydroxyphenyl)-5H-indeno[1,2-b]pyridin-5-ones. Bioorg. Chem. 2018, 81, 433439.

(27) Sasaki, S.; Drummen, G. P. C.; Konishi, G.-I. Recent Advances in Twisted Intramolecular Charge Transfer (TICT) Fluorescence and Related Phenomena in Materials Chemistry. J. Mater. Chem. C 2016, 4, 2731-2743.

(28) Wang, J.; Gu, X.; Zhang, P.; Huang, X.; Zheng, X.; Chen, M.; Feng, H.; Kwok, R. T. K.; Lam, J. W. Y.; Tang, B. Z. Ionization and Anion- $\pi^{+}$Interaction: ANew Strategy for Structural Design of Aggregation-Induced Emission Luminogens. J. Am. Chem. Soc. 2017, 139, 16974-16979.

(29) Zhu, C.; Yang, Q.; Liu, L.; Lv, F.; Li, S.; Yang, G.; Wang, S. Multifunctional Cationic Poly(p-phenylene vinylene) Polyelectrolytes for Selective Recognition, Imaging, and Killing of Bacteria Over Mammalian Cells. Adv. Mater. 2011, 23, 48054810.

(30) Davies, M. J. Singlet Oxygen-Mediated Damage to Proteins and Its Consequences. Biochem. Biophys. Res. Commun. 2003, 305, 761-770.

(31) Schweitzer, C.; Schmidt, R. Physical Mechanisms of Generation and Deactivation 
of Singlet Oxygen. Chem. Rev. 2003, 103, 1685-1757.

(32) Guo, L.; Niu, G.; Zheng, X.; Ge, J.; Liu, W.; Jia, Q.; Zhang, P.; Zhang, H.; Wang, P. Single Near-Infrared Emissive Polymer Nanoparticles as Versatile Phototheranostics. Adv. Sci. 2017, 4, 1700085.

(33) Gandin, E.; Lion, Y.; Vorst, A. V. D. Quantum Yield of Singlet Oxygen Production by Xanthene Derivatives. Photochem. Photobiol. 1983, 37, 271-278.

(34) Burnaugh, L.; Sabeur, K.; Ball, B. A. Generation of Superoxide Anion by Equine Spermatozoa as Detected by Dihydroethidium. Theriogenology 2007, 67, 580-589.

(35) Li, M.; Xia, J.; Tian, R.; Wang, J.; Fan, J.; Du, J.; Long, S.; Song, X.; Foley, J. W.;

Peng, X. Near-Infrared Light-Initiated Molecular Superoxide Radical Generator: Rejuvenating Photodynamic Therapy against Hypoxic Tumors. J. Am. Chem. Soc. 2018, $140,14851-14859$.

(36) Favor, J. D. L.; Anderson, E. J.; Hickner, R. C. Novel Method for Detection of Reactive Oxygen Species in vivo in Human Skeletal Muscle. Physiol. Res. 2014, 63, 387-392.

(37) Xu, S.; Yuan, Y.; Cai, X.; Zhang, C.; Hu, F.; Liang, J.; Zhang, G.; Zhang, D.; Liu, B. Tuning the Singlet-Triplet Energy Gap: A Unique Approach to Efficient Photosensitizers with Aggregation-Induced Emission (AIE) Characteristics. Chem. Sci. 2015, 6, 5824-5830.

(38) Lenthe, E. V.; Ehlers, A.; Baerends, E. J. Geometry Optimizations in the Zero Order Regular Approximation for Relativistic Effects. J. Chem. Phys. 1999, 110, 89438953.

(39) Schmidt, K.; Brovelli, S.; Coropceanu, V.; Beljonne, D.; Cornil, J.; Bazzini, C.; Caronna, T.; Tubino, R.; Meinardi, F.; Shuai, Z.; Brédas, J. L. Intersystem Crossing Processes in Nonplanar Aromatic Heterocyclic Molecules. J. Phys. Chem. A 2007, 111, 10490-10499.

(40) Xiong, Y.; Zhao, Z.; Zhao, W.; Ma, H.; Peng, Q.; He, Z.; Zhang, X.; Chen, Y.; He, X.; Lam, J. W. Y.; Tang, B. Z. Designing Efficient and Ultralong Pure Organic RoomTemperature Phosphorescent Materials by Structural Isomerism. Angew. Chem. Int. Ed. 2018, 57, 7997-8001. 
(41) Yang, L.; Wang, X.; Zhang, G.; Chen, X.; Zhang, G.; Jiang, J. AggregationInduced Intersystem Crossing: A Novel Strategy for Efficient Molecular Phosphorescence. Nanoscale 2016, 8, 17422-17426.

(42) Zhang, J.; Chen, W.; Chen, R.; Liu, X. K.; Xiong, Y.; Kershaw, S. V.; Rogach, A. L.; Adachi, C.; Zhang, X.; Lee, C. S. Organic Nanostructures of Thermally Activated Delayed Fluorescent Emitters with Enhanced Intersystem Crossing as Novel MetalFree Photosensitizers. Chem. Commun. 2016, 52, 11744-11747.

(43) Gui, C.; Zhao, E.; Kwok, R. T. K.; Leung, A. C. S. Lam, J. W. Y.; Jiang, M.; Deng, H.; Cai, Y.; Zhang, W.; Su, H.; Tang, B. Z. AIE-Active Theranostic System: Selective Staining and Killing of Cancer Cells. Chem. Sci. 2017, 8, 1822-1830.

(44) Li, J.; Kwon, N.; Jeong, Y.; Lee, S.; Kim, G.; Yoon, J. Aggregation-Induced Fluorescence Probe for Monitoring Membrane Potential Changes in Mitochondria. ACS Appl. Mater. Interfaces 2018, 10, 12150-12154. 